University of Northern lowa

UNI ScholarWorks

Faculty Publications

Faculty Work

$5-2018$

\title{
Out-of-plane electron transport in finite layer MoS2
}

\author{
R Holzapfel \\ Jake Weber \\ University of Northern lowa \\ See next page for additional authors
}

Let us know how access to this document benefits you

Copyright @Holzapfel, et al.

Follow this and additional works at: https://scholarworks.uni.edu/phy_facpub

Part of the Physics Commons

\section{Recommended Citation}

Holzapfel, R; Weber, Jake; Lukashev, Pavel; and Stollenwerk, Andrew, "Out-of-plane electron transport in finite layer MoS2" (2018). Faculty Publications. 28.

https://scholarworks.uni.edu/phy_facpub/28

This Article is brought to you for free and open access by the Faculty Work at UNI ScholarWorks. It has been accepted for inclusion in Faculty Publications by an authorized administrator of UNI ScholarWorks. For more information, please contact scholarworks@uni.edu. 
Authors

R Holzapfel, Jake Weber, Pavel Lukashev, and Andrew Stollenwerk 


\section{Out-of-plane electron transport in finite layer $\mathrm{MoS}_{2}$}

Cite as: J. Appl. Phys. 123, 174303 (2018); https://doi.org/10.1063/1.5026397

Submitted: 19 February 2018 . Accepted: 16 April 2018 . Published Online: 01 May 2018

R. Holzapfel, J. Weber, P. V. Lukashev, and A. J. Stollenwerk
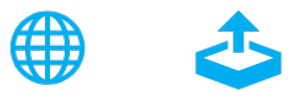

View Online

Export Citation

CrossMark

\section{ARTICLES YOU MAY BE INTERESTED IN}

Gate-tunable quantum dot in a high quality single layer $\mathrm{MoS}_{2}$ van der Waals heterostructure Applied Physics Letters 112, 123101 (2018); https://doi.org/10.1063/1.5021113

Improvement of gas-adsorption performances of Ag-functionalized monolayer $\mathrm{MoS}_{2}$ surfaces: A first-principles study

Journal of Applied Physics 123, 175303 (2018); https://doi.org/10.1063/1.5022829

Controlled synthesis of $2 \mathrm{D} \mathrm{MX} 2(\mathrm{M}=\mathrm{Mo}, \mathrm{W} ; \mathrm{X}=\mathrm{S}, \mathrm{Se})$ heterostructures and alloys Journal of Applied Physics 123, 204304 (2018); https://doi.org/10.1063/1.5025710

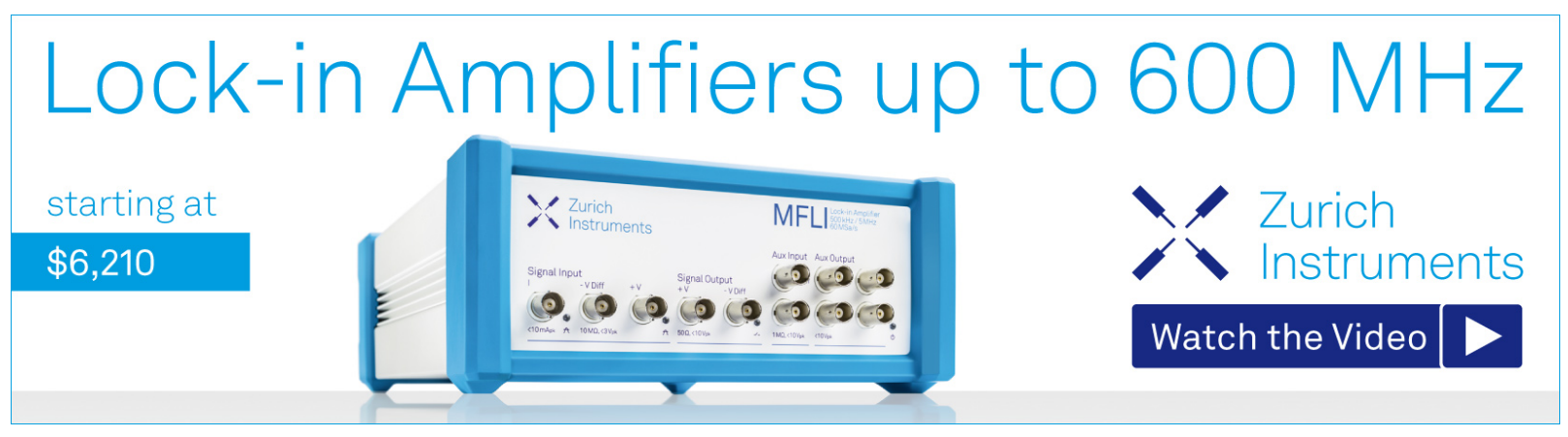




\title{
Out-of-plane electron transport in finite layer $\mathrm{MoS}_{2}$
}

\author{
R. Holzapfel, J. Weber, P. V. Lukashev, and A. J. Stollenwerk ${ }^{\text {a) }}$ \\ Department of Physics, University of Northern Iowa, 215 Begeman Hall, Cedar Falls, Iowa 50614-0150, USA
}

(Received 19 February 2018; accepted 16 April 2018; published online 1 May 2018)

\begin{abstract}
Ballistic electron emission microscopy (BEEM) has been used to study the processes affecting electron transport along the [0001] direction of finite layer $\mathrm{MoS}_{2}$ flakes deposited onto the surface of $\mathrm{Au} / \mathrm{Si}(001)$ Schottky diodes. Prominent features present in the differential spectra from the $\mathrm{MoS}_{2}$ flakes are consistent with the density of states of finite layer $\mathrm{MoS}_{2}$ calculated using density functional theory. The ability to observe the electronic structure of the $\mathrm{MoS}_{2}$ appears to be due to the relatively smooth density of states of $\mathrm{Si}$ in this energy range and a substantial amount of elastic or quasi-elastic scattering along the $\mathrm{MoS}_{2} / \mathrm{Au} / \mathrm{Si}(001)$ path. Demonstration of these measurements using BEEM suggests that this technique could potentially be used to study electron transport through van der Waals heterostructures, with applications in a number of electronic devices. Published by AIP Publishing. https://doi.org/10.1063/1.5026397
\end{abstract}

\section{INTRODUCTION}

Bulk $\mathrm{MoS}_{2}$ is a layered semiconductor that can be exfoliated into two-dimensional crystals. Recently, $\mathrm{MoS}_{2}$ has experienced a surge in activity due to the high in-plane electron mobility ${ }^{1,2}$ and direct band gap $^{3}$ associated with its finite layer form. These properties make it potentially useful in a broad range of devices such as field effect transistors (FETs), ${ }^{4,5}$ light emitting diodes, ${ }^{6}$ and solar cells. ${ }^{7}$ In most cases, electronic signals are sent and received along directions perpendicular to the plane using metallic contacts. As $\mathrm{MoS}_{2}$ based devices reach the goal of nanometer scale dimensions, the relative contribution of the contact to the overall performance increases significantly. ${ }^{8}$ In order to fully realize the intrinsic properties of nanometer scale $\mathrm{MoS}_{2}$, it is necessary to optimize the properties of the electrical contacts. Metal/semiconductor interfaces are typically investigated using techniques that measure average properties over large areas, such as current-voltage curves ${ }^{9}$ or internal photoemission spectroscopy. ${ }^{10}$ However, it is desirable to probe the interface on the nanometer scale.

The electronic structure can be measured with atomic resolution using a scanning tunneling microscope (STM), but this technique is most sensitive to surface states. Subsurface electronic properties of metal/semiconductor interfaces can be studied with nanometer precision by modifying an STM to perform ballistic electron emission microscopy (BEEM). ${ }^{11,12}$ In this three terminal setup, electrons from an STM tip (emitter) are injected into a grounded metal thin film (base) that forms a Schottky contact with a semiconductor (collector) substrate. Due to interactions in the metal and at the metal/ semiconductor interface, most of these electrons are detected as the STM current by the topside contact. Only a small fraction of these electrons travel ballistically through the metal with the sufficient energy to overcome the Schottky barrier and reach the backside contact. Assuming transverse momentum is conserved at the metal/semiconductor interface, these

a)andrew.stollenwerk@uni.edu electrons can only enter the semiconductor when there is an overlap between the distribution of electrons in the metal and the available states in the semiconductor. ${ }^{13}$ However, if momentum conservation fails due to isotropic elastic scattering at the interface, then all states in the semiconductor will be accessed and the resulting differential spectra will be proportional to the density of states (DOS) of the semiconductor substrate. ${ }^{14,15}$ A strong sensitivity to the interfacial electronic properties and scattering makes BEEM a powerful tool to analyze the electron transport between materials on the nanometer scale.

In this paper, we demonstrate that BEEM can be used to study out-of-plane electron transport in ultra-thin $\mathrm{MoS}_{2}$ flakes deposited onto the surface of an $\mathrm{Au} / \mathrm{Si}(001)$ Schottky diode. The first derivative of spectra from $\mathrm{MoS}_{2}$ exhibit distinct features not found in spectra from $\mathrm{Au} / \mathrm{Si}(001)$ alone, indicating that they originate from the electronic properties of $\mathrm{MoS}_{2}$. Additionally, these features have a strong resemblance with the DOS of finite layer $\mathrm{MoS}_{2}$ sheets calculated using the density functional theory (DFT). A poor overlap of states between the $\mathrm{MoS}_{2}$ and $\mathrm{Si}$ indicates substantial elastic scattering in the samples.

\section{METHODS}

\section{A. Experimental techniques}

Finite layer flakes of $\mathrm{MoS}_{2}$ were fabricated from powdered $\mathrm{MoS}_{2}$, 300-mesh 99.9\% molybdenum (IV) disulfide, using liquid exfoliation. ${ }^{16}$ The powder was first ball milled before being added to a $400 \mathrm{~mL}$ Berzelius beaker filled with isopropyl alcohol $(99.95 \%)$ at a concentration of approximately $6.0 \mathrm{~g} / \mathrm{L}$. This solution was sonicated at $20 \mathrm{kHz}$ using a Vibracell VDX-505 with a solid titanium alloy probe. A home-built cooling system consisting of an outer-beaker water jacket fitted with a copper radiator coil was used to draw excess heat from the solution by circulating $4{ }^{\circ} \mathrm{C}$ water through the copper tubing. The sonicator subjected this mixture to ultrasonic cavitation until $220 \mathrm{~kJ}$ of energy had been transferred to the solution. This amount was found to be 
large enough to break apart the $\mathrm{MoS}_{2}$ without forming carbon nanostructures in the process. ${ }^{17} \mathrm{~A}$ pulse mode was chosen for cavitation with a $75 \%$ duty cycle operating at $500 \mathrm{~W}$. The sonicated mixture was centrifuged in a Fisher Scientific accuSpin Micro 17 in order to extract the smaller particles found in the supernatant. One solution was created by centrifuging the sonicated mixture at $3 \mathrm{~g}$ for $5 \mathrm{~min}$, and the second at $5 \mathrm{~g}$ for $15 \mathrm{~min}$. The resulting suspensions were optically analyzed for absorbance properties using an Avaspec 2048 USB spectrometer, illuminated with an AvaLight DHc combination deuterium-hydrogen LED source. Background subtractions were used to remove signatures of the isopropanol.

BEEM samples consisted of $4 \mathrm{~nm}$ Au thin films thermally deposited on phosphorous-doped $\mathrm{Si}(001)$ wafers prepared similarly as in previous experiments. ${ }^{18}$ Deposition rates did not exceed $0.2 \AA / s$ as measured in situ using a quartz microbalance calibrated against an atomic force microscope. Chamber pressure did not exceed $6 \times 10^{-8}$ mbar during deposition. Finished samples were electrically and mechanically mounted to the sample holder ex situ using conductive silver paste. A drop cast method was used to deposit the $\mathrm{MoS}_{2}$ flakes onto the surface of the $\mathrm{Au}$ film. After the isopropanol alcohol evaporated, an electrical contact was made to the Au film using a BeCu clip. Immediately after this, the sample was inserted into an STM/BEEM chamber with a base pressure of $3 \times 10^{-9}$ mbar. Ballistic electron emission spectroscopy was obtained by positioning the tip directly above an $\mathrm{MoS}_{2}$ flake as shown in Fig. 1. Spectroscopy was acquired after stabilizing the tip above the sample with a tunneling bias of $0.8 \mathrm{~V}$ and a current set point of 20-100 nA. Differential spectra were numerically calculated from this data after smoothing with a window no greater than $50 \mathrm{meV}$. Tips were electrochemically etched from $0.25 \mathrm{~mm}$ polycrystalline tungsten wire in a $5 \mathrm{~mol} \mathrm{KOH}$ solution with a $5 \mathrm{~V}_{r m s}$ bias.

\section{B. Computational methods}

Density functional calculations were performed using the projector augmented-wave method (PAW), ${ }^{19}$ implemented in the Vienna ab initio simulation package (VASP) ${ }^{20}$ within the generalized-gradient approximation (GGA). ${ }^{21}$ The integration method ${ }^{22}$ with a $0.05 \mathrm{eV}$ width of smearing was used along with the plane-wave cut-off energy of $500 \mathrm{eV}$.

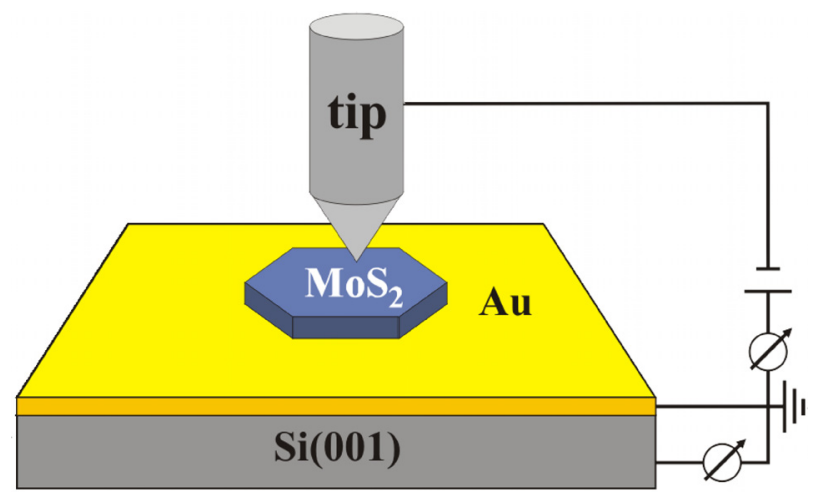

FIG. 1. Schematic of the experimental setup.
The convergence criteria were $10^{-5} \mathrm{eV}$ for atomic relaxation and $10^{-3} \mathrm{meV}$ for the total energy and electronic structure calculations. A k-point mesh of $12 \times 12 \times 2$ was used for the Brillouin zone integration in thin film geometry. The band structures were calculated along the following symmetry points in the Brillouin zone: $\Gamma(0,0,0), \Sigma(0.25,0,0), \mathrm{M}(0.5$, $0,0), \mathrm{K}(0.33,0.33,0), \Lambda(0.167,0.167,0)$, and $\Gamma(0,0,0)$. For all calculations, the lattice parameters were fully optimized to obtain equilibrium structures. Periodic boundary conditions were imposed in all calculations. A $20 \AA$ vacuum layer was added to the cell in the $\mathrm{z}$-direction to account for the periodic boundary condition in thin film geometry. Some of the results were obtained using the MedeA ${ }^{\circledR}$ software environment. $^{44}$

\section{RESULTS AND DISCUSSION}

The effects of ultrasonic agitation could be observed visually as the gray-black powder of the $\mathrm{MoS}_{2}$ suspension changed to a yellow-green hue. Absorption measurements of both suspensions are shown in Fig. 2 and are normalized to absorption at $4 \mathrm{eV}$ for comparison. Both spectra are characteristic of $\mathrm{MoS}_{2}$ with prominent peaks at approximately $1.85 \mathrm{eV}$ (A) and $2.03 \mathrm{eV}$ (B) corresponding to direct exciton transitions at the $\mathrm{K}$ point. ${ }^{23,24}$ The peak at about $2.8 \mathrm{eV}$ (C) is shifted considerably to a higher energy compared to the bulk value, indicating the presence of 1-2 monolayer (ML) flakes of $\mathrm{MoS}_{2}$ in solution. ${ }^{25}$ When applied to the Au film, a ring of material emerged along the perimeter of the drop as the isopropanol evaporated. No flakes were found when scanning toward the center of the ring, while the ring itself generally had a random clumping of material. Optimal locations to find isolated $\mathrm{MoS}_{2}$ flakes lying horizontal to the surface were typically found about ten microns from the inner side of the ring. Although no single monolayer $\mathrm{MoS}_{2}$ could be identified with certainty in the topographic images, bilayer flakes were present, typically with lateral dimensions in the order of $100 \mathrm{~nm}$. Larger flakes could reach thicknesses of $50 \mathrm{~nm}$ and were several microns in size.

Spectroscopy measured directly on the Au film is similar to the previous results ${ }^{18,26,27}$ and exhibits an average threshold voltage of $0.82 \pm 0.03 \mathrm{eV}$ as determined by a Bell-Kaiser

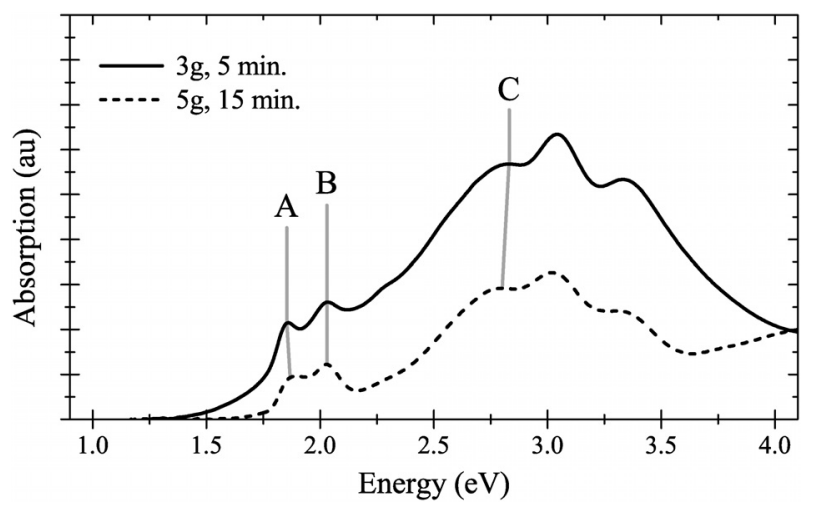

FIG. 2. Optical absorption measurements of $\mathrm{MoS}_{2}$ suspensions centrifuged at $3 \mathrm{~g}$ for $5 \mathrm{~min}$. (solid) and $5 \mathrm{~g}$ for $15 \mathrm{~min}$ (dashed) following $220 \mathrm{~kJ}$ of ultrasonic agitation. Exciton peaks characteristic of $\mathrm{MoS}_{2}$ are labeled as A, $\mathrm{B}$, and C. Spectra are normalized to absorption at $4 \mathrm{eV}$ for comparison. 
fit. ${ }^{11}$ Transmission through the $\mathrm{MoS}_{2}$ is reduced by at least two orders of magnitude as compared to the Au alone and is found to vary from point to point on the same flake. This is likely the result of a tunneling barrier at the $\mathrm{Au} / \mathrm{MoS}_{2}$ interface with a varying width due to the surface roughness (RMS $0.8 \pm 0.1 \mathrm{~nm}$ ) of the $\mathrm{Au}$, suppressing transmission in some locations. No signal could be detected on flakes with thickness greater than $8 \mathrm{~nm}$. Data from the $\mathrm{MoS}_{2}$ are characterized by the presence of several prominent peaks in the first derivative of the spectra as exemplified in Figs. 3(a) and 3(b) for a 2 and $8 \mathrm{ML}$ flake, respectively. The threshold voltages of these spectra are similar to those from the Au/Si samples, indicating that the $\mathrm{MoS}_{2} / \mathrm{Au}$ Schottky barrier must be less
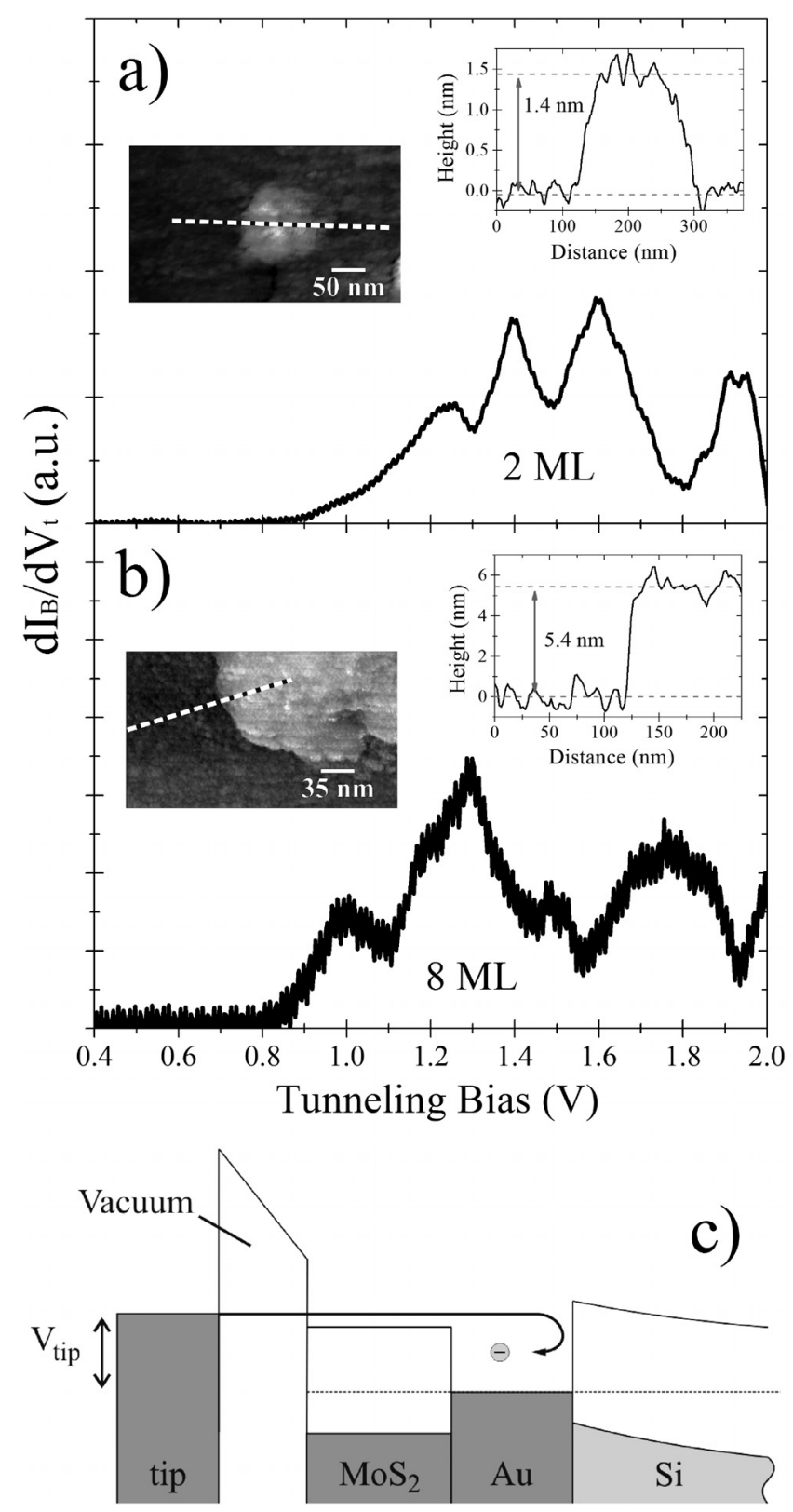

FIG. 3. Differential spectra from a $2 \mathrm{ML}$ (a) and $8 \mathrm{ML}$ (b) $\mathrm{MoS}_{2}$ flake on $\mathrm{Au} / \mathrm{Si}(001)$. Insets: STM images and line profiles of the $\mathrm{MoS}_{2}$ flakes for each corresponding spectrum. (c) Energy-band diagram for $\mathrm{MoS}_{2} / \mathrm{Au} /$ $\mathrm{Si}(001)$ showing that it is possible for electrons to have sufficient energy for tunneling into the conduction band of $\mathrm{MoS}_{2}$, but insufficient energy to enter the Si. than or equal to the barrier at the $\mathrm{Au} / \mathrm{Si}$ interface. In this configuration, electrons injected near the conduction band minimum $(\mathrm{CBM})$ of $\mathrm{MoS}_{2}$ are blocked by the Schottky barrier at the $\mathrm{Au} / \mathrm{Si}$ interface [see Fig. 3(c)], making it impossible to directly measure the $\mathrm{MoS}_{2}$ Schottky barrier height.

The Schottky-Mott rule predicts the Schottky barrier height $\left(\phi_{S B}\right)$ based on the work function of the metal $\left(\phi_{m}\right)$ and the electron affinity of the semiconductor $\left(\chi_{s c}\right)$, $\phi_{S B}=\phi_{m}-\chi_{s c}$. Accordingly, the Schottky barrier between $\mathrm{Au}$ and bulk $\mathrm{MoS}_{2}$ is expected to be approximately $0.90 \mathrm{eV},{ }^{28}$ but is found to be lower than this experimentally due to Fermi level pinning near the conduction band. Computational studies suggest global pinning results from both dipole formation and the introduction of gap states at the interface. ${ }^{29}$ Localized pinning has been observed experimentally in the vicinity of subsurface metal-like defects. ${ }^{30}$ The relatively low density of these defects accounts for the conflicting reports of barrier heights found in literature, which range from 0.06 to $0.62 \mathrm{eV}^{9,28,31,32}$ and, in some cases, are found to be ohmic. ${ }^{1}$ Because transmission occurs over large areas, values extracted from standard IV curves are dominated by sites with lower barrier heights. BEEM spectra from $\mathrm{Au}$ on bulk $\mathrm{MoS}_{2}$ yield higher Schottky barriers $(0.48-0.62 \mathrm{eV}){ }^{31}$ most likely at locations without the subsurface defects.

The origin of the structure in the spectra is attributed to the presence of $\mathrm{MoS}_{2}$ since spectra on the $\mathrm{Au} / \mathrm{Si}(001)$ alone are featureless in this energy range. Distinct peaks in the spectra could result from resonant tunneling behavior, previously observed in finite layer $\mathrm{MoS}_{2}{ }^{33}$ However, this is unlikely to be the case here as the spacing of the peaks in our data does not increase with confinement as would be expected. To gain better insight into the nature of these peaks, we examine the electronic structure of finite layer $\mathrm{MoS}_{2}$. Ignoring the effects of the van der Waals interaction with $\mathrm{Au},{ }^{34}$ the band structure and DOS for 2 and $8 \mathrm{ML} \mathrm{MoS}_{2}$ were calculated using DFT and are plotted in Figs. 4(a) and 4(b). For comparison, the experimental data (grey) are superimposed on the DOS (black). The alignment of the numerical results to the data was made using a least squares fit to the four prominent peaks in the data after assuming the CBM of $8 \mathrm{ML} \mathrm{MoS}_{2}$ to be similar to the bulk value. ${ }^{31}$ The alignment to the $2 \mathrm{ML}$ data was determined in a similar fashion. However, the CBM of the $2 \mathrm{ML}$ data was assumed to be shifted toward higher energies to account for a $0.18 \mathrm{eV}$ increase relative to the bulk. ${ }^{35}$ These fits place the CBM of the $8 \mathrm{ML}$ flake at $0.62 \mathrm{eV}$ and the $2 \mathrm{ML}$ flake at $0.79 \mathrm{eV}$ above the Fermi level. The strong resemblance between the numerical and experimental data is not necessarily surprising. Using the well-established theory of STM, electrons tunneling into $\mathrm{MoS}_{2}$ will have an energy distribution that is largely shaped by the DOS of the $\mathrm{MoS}_{2}$ given that the $\mathrm{W}$ tip has a relatively flat density of states. ${ }^{36}$ However, to reach the backside contact, there must be matching states present in the $\mathrm{Si}$.

The projection of the Si Brillouin zone (square) onto the (001) plane is superimposed onto that of $\mathrm{MoS}_{2}(0001)$ (hexagon) in Fig. 4(c). The Si CBM is represented by the +symbol with constant energy pockets depicted at $0.2 \mathrm{eV}$ 


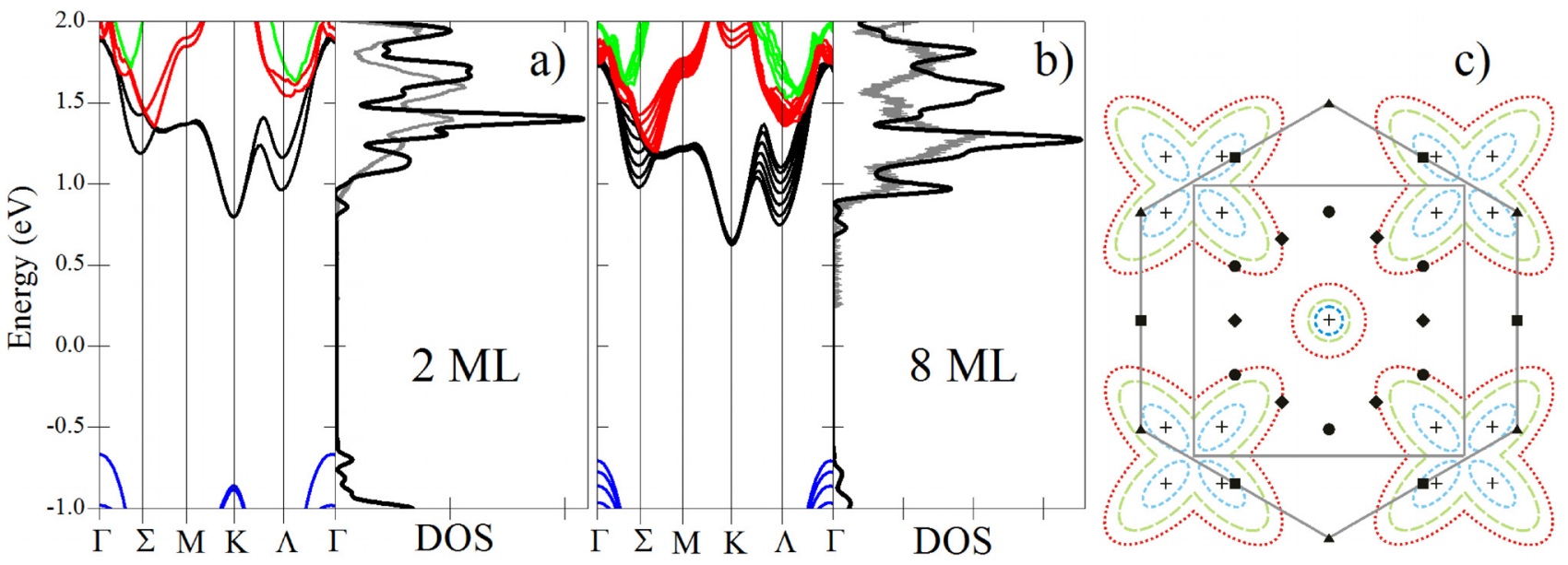

FIG. 4. Band structure and DOS calculations for $2 \mathrm{ML}$ (a) and $8 \mathrm{ML}$ (b) sheets of $\mathrm{MoS}_{2}$. The experimental data (grey) are superimposed on the DOS (black) for comparison. (c) Projected $\mathrm{Si}(001)$ Brillouin zone (square) onto the $\mathrm{MoS}_{2}(0001)$ Brillouin zone. The points of symmetry associated with $\mathrm{MoS}_{2}$ are represented by the symbols $\triangle(\mathrm{K}), \bigcirc(\Lambda), \square(\mathrm{M})$, and $\diamond(\Sigma)$. Constant energy pockets are depicted at $0.2 \mathrm{eV}$ (short-dashed blue line), $0.45 \mathrm{eV}$ (dashed green line), and $1.0 \mathrm{eV}$ (dotted red line) above the CBM.

(short-dashed blue line), $0.45 \mathrm{eV}$ (dashed green line), and $1.0 \mathrm{eV}$ (dotted red line) above the CBM. The points of symmetry associated with $\mathrm{MoS}_{2}$ are represented by the symbols $\triangle(\mathrm{K}), \bigcirc(\Lambda), \square(\mathrm{M})$, and $\diamond(\Sigma)$. Accounting for the random orientation of an $\mathrm{MoS}_{2}$ flake, there is a poor overlap of states regardless of the alignment between the two materials up to energies of about $0.45 \mathrm{eV}$ above the CBM of Si. It is not until about $1.0 \mathrm{eV}$ above the $\mathrm{CBM}$ when the overlap becomes orientation independent. In order to observe the DOS of the $\mathrm{MoS}_{2}$ in the BEEM current, the electrons must undergo sufficient elastic scattering to access the states in $\mathrm{Si}$, but experience minimal inelastic scattering to maintain the energy distribution. Considering the surface roughness associated with a thermally evaporated Au film, ${ }^{18}$ disorder at the $\mathrm{MoS}_{2} /$ $\mathrm{Au}$ interface is expected to act as a source of quasi-elastic scattering, broadening the distribution of transverse momentum. Further broadening occurs due to quasi-elastic scattering at the $\mathrm{Au} / \mathrm{Si}$ interface, all of which is amplified by the reflections between the $\mathrm{MoS}_{2}$ and $\mathrm{Si}^{37}$ Little change to the energy distribution is expected as inelastically scattered electrons in the Au will be filtered by the Schottky barrier ${ }^{37}$ and impact ionization in the $\mathrm{Si}$ is negligible at these energies. ${ }^{38}$ Thus, the final energy distribution of electrons entering the Si substrate will depend primarily on the relative DOS of the $\mathrm{MoS}_{2}$ and the Si. The relatively smooth DOS of $\mathrm{Si}^{39}$ in this energy range allows the distinct features of the $\mathrm{MoS}_{2}$ to display prominently.

\section{CONCLUSIONS}

We have shown that BEEM can be used to investigate out-of-plane electron transport in finite layer $\mathrm{MoS}_{2}$ deposited onto the surface of $\mathrm{Au} / \mathrm{Si}(001)$. By itself, this is remarkable given that the $\mathrm{MoS}_{2}$ flakes were created using liquid exfoliation and deposited using a drop cast method. Differential spectra from these samples exhibit the convoluted DOS of $\mathrm{MoS}_{2}$ and Si with specific features from the $\mathrm{MoS}_{2}$ easily distinguished due to the relatively smooth DOS of $\mathrm{Si}$ in this energy range. These results suggest that electrons tunneling into the $\mathrm{MoS}_{2}$ undergo a considerable amount of elastic or quasi-elastic scattering events in order to access states in the Si. The strong match between the features in the spectra and the calculated DOS indicates minimal inelastic scattering. This experimental technique could potentially be used to study out-of-plane transport through van der Waals heterostructures with applications in devices such as the vertical tunneling FET, ${ }^{40,41}$ photo-detectors ${ }^{42}$ and solar cells. ${ }^{43}$

\section{ACKNOWLEDGMENTS}

This work was supported by the National Science Foundation Grant No. DMR-1410496. The authors would like to thank T. E. Kidd and L. H. Strauss for technical support with the sonicator and ball miller.

${ }^{1}$ B. Radisavljevic, A. Radenovic, J. Brivio, V. Giacometti, and A. Kis, Nat. Nanotechnol. 6, 147 (2011).

${ }^{2}$ S. Kim, A. Konar, W.-S. Hwang, J. H. Lee, J. Lee, J. Yang, C. Jung, H. Kim, H. Kim, J.-B. Yoo, J.-Y. Choi, Y. W. Jin, S. Y. Lee, D. Jena, W. Choi, and K. Kim, Nat. Commun. 3, 1011 (2012).

${ }^{3}$ K. F. Mak, C. Lee, J. Hone, J. Shan, and T. F. Heinz, Phys. Rev. Lett. 105, 136805 (2010).

${ }^{4}$ F. M. Espinosa, Y. K. Ryu, K. Marinov, D. Dumcenco, A. Kis, and R. Garcia, Appl. Phys. Lett. 106, 103503 (2015).

${ }^{5}$ W. Wu, D. De, S.-C. Chang, Y. Wang, H. Peng, J. Bao, and S.-S. Pei, Appl. Phys. Lett. 102, 142106 (2013).

${ }^{6}$ O. Lopez-Sanchez, E. Alarcon Llado, V. Koman, A. Fontcuberta i Morral, A. Radenovic, and A. Kis, ACS Nano 8, 3042 (2014).

${ }^{7}$ O. Salehzadeh, M. Djavid, N. H. Tran, I. Shih, and Z. Mi, Nano Lett. 15, 5302 (2015).

${ }^{8}$ C. D. English, G. Shine, V. E. Dorgan, K. C. Saraswat, and E. Pop, Nano Lett. 16, 3824 (2016).

${ }^{9}$ N. Kaushik, A. Nipane, F. Basheer, S. Dubey, S. Grover, M. M. Deshmukh, and S. Lodha, Appl. Phys. Lett. 105, 113505 (2014).

${ }^{10}$ R. Mamy and X. Zaoui, Phys. Status Solidi B 156, K109 (1989).

${ }^{11}$ W. J. Kaiser and L. D. Bell, Phys. Rev. Lett. 60, 1406 (1988).

${ }^{12}$ L. D. Bell and W. J. Kaiser, Phys. Rev. Lett. 61, 2368 (1988).

${ }^{13}$ P. de Andres, F. Garcia-Vidal, K. Reuter, and F. Flores, Prog. Surf. Sci. 66, 3 (2001).

${ }^{14}$ R. Ludeke, Phys. Rev. Lett. 70, 214 (1993).

${ }^{15}$ A. Chahboun, R. Coratger, F. Ajustron, J. Beauvillain, I. M. Dharmadasa, and A. P. Samantilleke, J. Appl. Phys. 87, 2422 (2000).

${ }^{16}$ J. N. Coleman, M. Lotya, A. O’Neill, S. D. Bergin, P. J. King, U. Khan, K. Young, A. Gaucher, S. De, R. J. Smith et al., Science 331, 568 (2011). 
${ }^{17}$ A. J. Stollenwerk, E. Clausen, M. Cook, K. Doore, R. Holzapfel, J. Weber, R. He, and T. E. Kidd, J. Nanosci. Nanotechnol. 18, 3171 (2018).

${ }^{18}$ M. W. Eckes, B. E. Friend, and A. J. Stollenwerk, J. Appl. Phys. 115, 163710 (2014).

${ }^{19}$ P. E. Blöchl, Phys. Rev. B 50, 17953 (1994).

${ }^{20}$ G. Kresse and D. Joubert, Phys. Rev. B 59, 1758 (1999).

${ }^{21}$ J. P. Perdew, K. Burke, and M. Ernzerhof, Phys. Rev. Lett. 77, 3865 (1996).

${ }^{22}$ M. Methfessel and A. Paxton, Phys. Rev. B 40, 3616 (1989).

${ }^{23}$ K. Wang, J. Wang, J. Fan, M. Lotya, A. O'Neill, D. Fox, Y. Feng, X. Zhang, B. Jiang, Q. Zhao et al., ACS Nano 7, 9260 (2013).

${ }^{24}$ A. Splendiani, L. Sun, Y. Zhang, T. Li, J. Kim, C.-Y. Chim, G. Galli, and F. Wang, Nano Lett. 10, 1271 (2010).

${ }^{25}$ K. P. Dhakal, D. L. Duong, J. Lee, H. Nam, M. Kim, M. Kan, Y. H. Lee, and J. Kim, Nanoscale 6, 13028 (2014).

${ }^{26}$ B. Friend, E. Wolter, T. Kidd, and A. Stollenwerk, Appl. Phys. Lett. 102, 091605 (2013).

${ }^{27}$ A. J. Stollenwerk, E. J. Spadafora, J. J. Garramone, R. J. Matyi, R. L. Moore, and V. P. LaBella, Phys. Rev. B 77, 033416 (2008).

${ }^{28}$ S. McDonnell, A. Azcatl, R. Addou, C. Gong, C. Battaglia, S. Chuang, K. Cho, A. Javey, and R. M. Wallace, ACS Nano 8, 6265 (2014).

${ }^{29}$ C. Gong, L. Colombo, R. M. Wallace, and K. Cho, Nano Lett. 14, 1714 (2014).

${ }^{30} \mathrm{P}$. Bampoulis, R. van Bremen, Q. Yao, B. Poelsema, H. J. W. Zandvliet, and K. Sotthewes, ACS Appl. Mater. Interfaces 9, 19278 (2017).
${ }^{31}$ M. Cook, R. Palandech, K. Doore, Z. Ye, G. Ye, R. He, and A. J. Stollenwerk, Phys. Rev. B 92, 201302 (2015).

${ }^{32}$ D. Qiu and E. K. Kim, Sci. Rep. 5, 13743 (2015).

${ }^{33}$ L.-N. Nguyen, Y.-W. Lan, J.-H. Chen, T.-R. Chang, Y.-L. Zhong, H.-T. Jeng, L.-J. Li, and C.-D. Chen, Nano Lett. 14, 2381 (2014).

${ }^{34}$ M. Farmanbar and G. Brocks, Phys. Rev. B 93, 085304 (2016).

${ }^{35}$ S.-L. Li, K. Komatsu, S. Nakaharai, Y.-F. Lin, M. Yamamoto, X. Duan, and K. Tsukagoshi, ACS Nano 8, 12836 (2014).

${ }^{36}$ H. Jansen and A. Freeman, Phys. Rev. B 30, 561 (1984).

${ }^{37}$ L. D. Bell, Phys. Rev. Lett. 77, 3893 (1996).

${ }^{38}$ A. Bauer and R. Ludeke, Phys. Rev. Lett. 72, 928 (1994).

${ }^{39}$ J. Rowe and H. Ibach, Phys. Rev. Lett. 31, 102 (1973).

${ }^{40}$ W. J. Yu, Z. Li, H. Zhou, Y. Chen, Y. Wang, Y. Huang, and X. Duan, Nat. Mater. 12, 246 (2013).

${ }^{41}$ T. Georgiou, R. Jalil, B. D. Belle, L. Britnell, R. V. Gorbachev, S. V. Morozov, Y.-J. Kim, A. Gholinia, S. J. Haigh, O. Makarovsky et al., Nat. Nanotechnol. 8, 100 (2013).

${ }^{42}$ M. Massicotte, P. Schmidt, F. Vialla, K. G. Schädler, A. Reserbat-Plantey, K. Watanabe, T. Taniguchi, K.-J. Tielrooij, and F. H. Koppens, Nat. Nanotechnol. 11, 42 (2016).

${ }^{43}$ F. Wang, Z. Wang, K. Xu, F. Wang, Q. Wang, Y. Huang, L. Yin, and J. He, Nano Lett. 15, 7558 (2015).

${ }^{44}$ Mede $A \circledR$ Version 2.19. MedeA ${ }^{\circledR}$ is a registered trademark of Materials Design, Inc. Angel Fire, New Mexico, USA. 